\title{
Alternativas de minimização de resíduos em uma INDÚSTRIA DE ALIMENTOS DA REGIÃO METROPOlitANA de CuRITIBA
}

\section{WASTE MINIMIZATION MEASURES IN A FOOD INDUSTRY LOCATED IN THE METROPOLITAN AREA OF CURITIBA}

\begin{abstract}
BÁRBARA ZANICOTTI LEITE
Engenheira de Alimentos pela Pontifícia Universidade Católica do Paraná. Mestre em Tecnologia Química no Programa de Pós-graduação em Tecnologia de Alimentos pela Universidade Federal do Paraná. Atuando na área de controle de poluição de Indústrias desde 1996. Pesquisadora da Assessoria de Desenvolvimento e Pesquisa da SANEPAR - Companhia de Saneamento do Paraná desde 2004

\section{URIVALD PAWLOWSKY}

Engenheiro Químico pela Universidade Federal do Paraná (UFPR). Mestre em Ciências pela COPPE - Universidade Federal do Rio de Janeiro (UFRJ). Doutor (PhD) pela State University of New York (USA). Professor Titular de Engenharia Ambiental das Engenharias Ambiental e Química da UFPR. Ex-diretor do Departamento do Meio Ambiente da SUREHMA. Consultor da Organização Pan-americana da Saúde, Ógãos governamentais e Indústrias
\end{abstract}

Recebido: 31/08/04 Aceito: 05/04/05

\section{RESUMO}

Este trabalho foi realizado em uma Indústria de Alimentos da Região Metropolitana de Curitiba. Esta técnica é baseada na redução da quantidade e/ou toxicidade dos poluentes gerados pela empresa e possui como premissa básica o controle da poluição na sua fonte. O trabalho foi desenvolvido em quatro etapas. A primeira constituiu da descrição da unidade industrial. $\mathrm{Na}$ segunda etapa fez-se o levantamento e a caracterização dos resíduos gerados pelo processo produtivo. Afim de saber com qual dos resíduos a minimização deveria ser iniciada realizou-se a priorização dos mesmos com o uso de um modelo matemático. Os resíduos de natureza orgânica, tanto das linhas de produção quanto da estação de tratamento de águas residuárias foram selecionados como prioritários. Por fim, a quarta etapa do trabalho constou da proposição de medidas de minimização. As principais estratégias adotadas foram: treinamento e conscientização de funcionários, controle dos ajustes nas variáveis de tempo, temperatura e vazão de equipamentos bem como a verificação das especificaçôes das matérias-primas no momento do recebimento. A minimização de resíduos é uma opção a ser seguida para o gerenciamento ambiental da Indústria de Alimentos, pois, além de reduzir os gastos com disposição e tratamento de resíduos, ainda aumenta a eficiência dos processos produtivos.

PALAVRAS-CHAVE: Minimização, resíduos, indústria de alimentos, gestão ambiental.

\begin{abstract}
This work was developed in a food industry located in the metropolitan area of Curitiba, Paraná State. It is based in a reduction of quantity andlor toxicity of the generated polutants in the factory and has as basis the pollution control in its source. If the reduction of the waste in its source is not possible, the recycling strategy would be used. This work was developed in four stages. The first stage was the description of the industrial plant. In the second stage was done the assessment and characteristics of the wastes. The third stage was the waste priorization with a mathematical model to obtain the order of minimization. The organic natural waste, even from the productive process as from the wastewather treatment plant, were selected as priorities. The fourth stage of the waste minimization programme was the proposition of the minimization measures. The main recomendations were:: training the employers, ajustment and control at the time of the equipments, temperature, flow, and raw material specification control at the time of reception. The waste minimization is a good option for the environmental management of the food industry because besides reducing costs with waste disposal and treatment, it also increases production process efficiency.
\end{abstract}

KEYWORDS: Minimization, waste, food industry, environmental management.

\section{INTRODUÇÃO}

A humanidade está usando $20 \%$ a mais de recursos naturais do que o planeta é capaz de repor, com isso está avançando nos estoques naturais da Terra, lem- brando daqueles recursos que não são renováveis, como o petróleo (Prado Filho, 2002).

Desta forma as empresas vêm sofrendo uma tendência mundial que é a crescente pressão para serem mais respon- sáveis e cuidadosas com o meio ambiente. Esta pressão vem do governo, consumidores, acionistas e financiadores, bem como de grupos não-governamentais (Tibor \& Feldman, 1996). Se uma empresa reduzir a quantidade de poluentes 
gerados no seu processo, então teremos uma maior preservação do meio ambiente, além da economia nos gastos relacionados à disposição final dos mesmos.

Em face ao progressivo aumento nos custos de disposição adequada e o baixo desempenho ambiental das medidas adotadas com relação aos resíduos industriais, as indústrias têm direcionado seus esforços para o desenvolvimento de soluçôes mais efetivas. Dentre elas encontrase a minimização de resíduos, um conjunto de medidas que visa antes de tudo a redução máxima da quantidade de resíduos gerada pelos processos industriais. Esta postura tem se mostrado bastante eficiente para combater o aumento da degradação do meio ambiente, além de atender às legislações, reduzir gastos e melhorar a imagem pública da empresa. Porém, uma das grandes dificuldades encontradas é a visão de que o resíduo é lixo, ou resto de um processo produtivo, e não matéria-prima e energia em potencial para outro tipo de processo (Matos \& Schalch, 2000; Pawlowsky, 2002). Esta visão deve ser mudada, para que empresas possam operar de maneira sustentável.

Para a implantação de medidas de prevenção à poluição é fundamental saber como a mesma ocorre, quais são suas fontes e que processos a geram (Mota, 1999). E como nenhum processo consegue operar com a eficiência de $100 \%$, sempre se tem a existência de resíduos, pois quando são fornecidos matéria-prima e insumos a um processo obtém-se os produtos e resíduos (Pawlowsky, 2002). Quanto maiores forem as perdas de matéria-prima e insumos de um processo, maior será a quantidade de resíduos líquidos, sólidos ou gasosos gerados. Assim, quando se tem o uso de toneladas de matérias-primas por dia, toneladas de resíduos são produzidos como resultado (Crittenden \& Kolaczkowski, 1995; Henningsson, Smith \& Hyde, 2001; Zbontar \& Glavic, 2000). Toda perda de matéria-prima, energia ou qualquer outro recurso natural representa uma ineficiência do processo e a geração de Resíduos (Miles, Munilla \& Mcclurg, 1999).

Depois de feito um levantamento e caracterização completos dos resíduos produzidos pela empresa, faz-se necessário a classificação dos mesmos. Com o resultado desta classificação a empresa pode saber qual é o resíduo prioritário, aquele com o qual a empresa deve se ocupar primeiramente. Cercal (2000), propõe um modelo matemático para realizar esta clas- sificação. O modelo trata dos aspectos de valor econômico, riscos à saúde e ambientais e do critério de facilidade de minimização dos resíduos.

O presente trabalho, é de grande utilidade para empresas que desejam melhorar o gerenciamento dos seus resíduos, por apresentar uma metodologia de minimização completa e com suas etapas detalhadas. Tem-se também que a preotodos os setores da sociedade, o respeito que uma empresa tem pelo meio ambiente está diretamente relacionado à sua aceitação pública. Uma empresa que deseja manter bons níveis de desempenho econômico deve aceitar que as tecnologias de fim de tubo não são as melhores opções de disposição final de resíduos e, sim, a prevenção. Ao invés de gerar poluentes e depois preocupar-se com a forma de dispô-los, deve-se evitar a sua geração. Neste ponto a minimização de resíduos focaliza o problema de modo a evitar que um determinado resíduo seja gerado ou seja gerado na menor quantidade possível, sem afetar a qualidade do produto final.

\section{METODOLOGIA}

As etapas de trabalho desenvolvidas foram: descrição da empresa, levantamento, caracterização, priorização de resíduos e proposição de alternativas de minimização.

\section{Descrição da empresa}

Na primeira etapa do trabalho foi feito um reconhecimento da unidade industrial. Este englobou aspectos tais como cupação ambiental é cada vez maior em

localização, produção, número de funcionários, tipos de matérias-primas utilizadas e também um conhecimento da visão da empresa a respeito do meio ambiente. A mesma foi realizada a fim de se conhecer o funcionamento industrial e de se dar início ao projeto de minimização de resíduos.

O trabalho foi desenvolvido em uma indústria de alimentos da Cidade Industrial de Curitiba que produz snacks extrusados fritos, extrusados assados e batatas fritas nas formas chips e palha. A empresa possui aproximadamente 300 funcionários, divididos em três turnos produtivos, operando cinco dias por semana.

Como o trabalho objetiva uma abordagem de todos os resíduos gerados no setor produtivo da empresa, foi estudada a planta baixa da mesma realizando-se uma divisão em setores, tomando-se como base para esta divisão as atividades desenvolvidas por cada setor. Desta forma, facilitouse a caracterização dos resíduos e o andamento do trabalho. A unidade industrial foi dividida em sete setores (Figura 1).

\section{Levantamento e caracterização de resíduos}

Foi feito o levantamento das fontes poluidoras, conhecendo-se o fluxograma de processo, as matérias-primas, insumos consumidos e produtos fabricados. $\mathrm{Na}$ caracterização das fontes poluidoras foi feita a localização de saídas de efluentes líquidos e sólidos, águas de processo, águas de utilidades, pluviais e esgoto sanitário. Foram medidas as vazões e foi feita a amostragem das diversas fontes de efluentes.

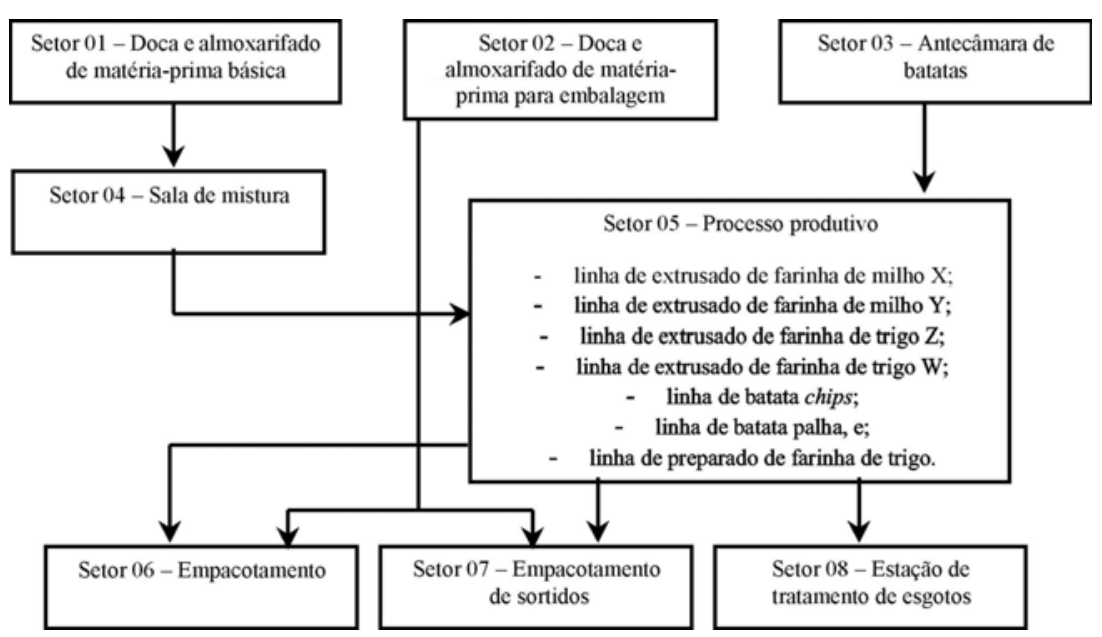

Figura I- Fluxograma simplificado de processo 


\section{Priorização dos resíduos}

A metodologia utilizada para a priorização dos resíduos é a proposta por Cercal (2000), e consiste num modelo matemático que analisa os aspectos de valor, risco e de facilidade de minimização do resíduo atribuindo a cada um destes aspectos uma classificação

O modelo proposto por Cercal (2000), considera "produtos" o conjunto de dados referentes a uma determinada situação de produção e "equipamen- tos" os objetos físicos por onde passam e/ou são processados e/ou tratados os materiais. Os resíduos são provenientes de um único ou vários equipamentos e possuem diferentes composiçóes e quantidades, e cada resíduo pode ter uma ou mais disposiçōes finais. O modelo matemático trata cada resíduo de acordo com as diferentes possibilidades de disposição final e analisa três aspectos básicos: análise do resíduos por valor, análise do resíduo por risco e análise do resíduo por facilidade de minimização.
Neste tópico o resíduo mais prioritário, ou seja, aquele que deve ser escolhido para se iniciar a minimização é o que apresentar o menor valor global. $\mathrm{O}$ modelo considera dados referentes à hierarquia de prioridades de minimização, a relação do resíduo com o processo (intrínseco, semi-intrínseco ou extrínseco) e se a geração é ou não constante (resíduo fixo ou variável). (Cercal, 2000).

A Tabela 1, contém a descrição das variáveis utilizadas no modelo matemático com suas respectivas identificações.

Tabela I - Variáveis do modelo matemático

\begin{tabular}{|c|c|}
\hline Natureza das variáveis & Siglas \\
\hline Quantidade total do resíduo & $\mathrm{W}_{\text {Total }}$ \\
\hline Número de produtos analisados simultaneamente & $\mathrm{p}$ \\
\hline Número de equipamentos onde o resíduo é gerado & e \\
\hline Número de materiais que compõem o resíduo & $\mathrm{m}$ \\
\hline Número de destinações finais dadas ao resíduo & $\mathrm{d}$ \\
\hline Classificação do resíduo conforme a periculosidade & $\Pi$ \\
\hline Relação do resíduo com o processo & $\Omega$ \\
\hline Valor unitário ${ }^{1}$ do material genérico "i" & $\$_{i}^{+2}$ \\
\hline Alteração percentual admissível para o valor do Material genérico "i" & $\Delta \$_{\mathrm{i}}^{3}{ }^{3}$ \\
\hline $\begin{array}{l}\text { Percentual do material genérico "i" na composição do resíduo gerado no } \\
\text { equipamento genérico "j", para o produto genérico "k" }\end{array}$ & $\mathrm{X}_{\mathrm{ijk}}$ \\
\hline $\begin{array}{l}\text { Percentual do total de resíduo gerado no equipamento genérico "j", para o produto } \\
\text { genérico "k", que sofre a disposição genérica "h" }\end{array}$ & $Y_{\text {hjk }}$ \\
\hline $\begin{array}{l}\text { Percentual do total do resíduo que é gerado no equipamento genérico "j", para o } \\
\text { produto genérico "k" }\end{array}$ & $\mathrm{Z}_{\mathrm{jk}}$ \\
\hline Percentual do total do resíduo gerado para o produto genérico "k" & $\mathrm{W}_{\mathrm{k}}$ \\
\hline $\begin{array}{l}\text { Custo unitário de beneficiamento do resíduo gerado no equipamento genérico "j", } \\
\text { para o produto genérico "k", que sofre a disposição genérica "h" }\end{array}$ & $\$_{\text {Bhjk }}^{-}$ \\
\hline $\begin{array}{l}\text { Custo unitário de transporte do resíduo gerado no equipamento genérico "j", para o } \\
\text { produto genérico "k", que sofre a disposição genérica "h" }\end{array}$ & $\$ \$_{\text {Thjk }}^{-}$ \\
\hline $\begin{array}{l}\text { Custo unitário de tratamento e disposição do resíduo gerado no equipamento } \\
\text { genérico "j", para o produto genérico "k", que sofre a disposição genérica "h" }\end{array}$ & $\$ \$_{\text {TDhjk }}^{-}$ \\
\hline $\begin{array}{l}\text { Custo unitário de geração e permanência do resíduo proveniente do equipamento } \\
\text { genérico "j", para o produto genérico "k", que sofre a disposição genérica "h" }\end{array}$ & $\$$ GPhjk \\
\hline $\begin{array}{l}\text { Retorno obtido por destinar o resíduo gerado no equipamento genérico "j", para o } \\
\text { produto genérico "k", à disposição genérica "h" }\end{array}$ & $\$_{\text {Rhjk }}^{+}$ \\
\hline Calcula $\$+?$ & $\mathrm{D}_{\$+}^{\mathrm{S} / \mathrm{N}}$ \\
\hline Calcula $\$_{B}^{-} ?$ & $\mathrm{D}_{\$ \mathrm{~B}}^{\mathrm{S} / \mathrm{N}}$ \\
\hline Calcula $\$_{\mathrm{T}}^{-} ?$ & $\mathrm{D}_{\$ \mathrm{~T}}^{\mathrm{S} / \mathrm{N}}$ \\
\hline
\end{tabular}

${ }^{1} \mathrm{O}$ termo unitário se refere a um quilograma, um metro cúbico, uma tonelada ou outra unidade qualquer de quantidade do material.

${ }^{2} \mathrm{O}$ símbolo “\$” indica valor monetário. O índice “+” representa ganho monetário, enquanto que o “-” indica prejuízo. 3 O símbolo " $\Delta$ ", anteposto a " $\$$ ”, indica alteração do valor, e o símbolo "\%", sobrescrito, indica que a alteração é percentual.

${ }^{4} \mathrm{O}$ retorno representa o ganho monetário obtido com a venda, reaproveitamento, reutilização ou reciclagem de uma unidade de quantificação do resíduo. 
Tabela I - Variáveis do modelo matemático (continuação)

\begin{tabular}{lcc}
\hline & Natureza das variáveis & Siglas \\
\hline Calcula $\$_{\mathrm{TD}}^{-}$? & $\mathrm{D}^{\mathrm{S} / \mathrm{N}-}$ \\
Calcula $\$_{\mathrm{GP}}^{-}$ & $\mathrm{D}^{\mathrm{S} / \mathrm{N}}{ }_{\$ \mathrm{GP}}$ \\
Calcula $\$_{\mathrm{R}}^{+}$? & $\mathrm{D}^{\mathrm{S} / \mathrm{N} \$_{\mathrm{R}}^{+}}$ \\
Valor unitário do resíduo & $\$^{+}$
\end{tabular}

Alteração percentual admissível para o valor unitário do resíduo

$\Delta \$^{\%}$

Custo unitário de beneficiamento do resíduo

$\$_{\text {B }}^{-}$

Custo unitário de transporte do resíduo

Custo unitário de tratamento e disposição do resíduo

$\$^{-}$

Custo unitário de geração e permanência do resíduo

$\${ }_{\mathrm{TD}}^{-}$

$\$_{\text {GP }}^{-}$

Retorno obtido conforme as disposições do resíduo

$\$_{\mathrm{R}}^{+}$

Valor unitário do resíduo não corrigido

$\$$

Índice de priorização hierárquica de minimização de resíduos (IPHMR)

Base do IPHMR, ponderada entre todos os equipamentos onde o resíduo é gerado

e para todos os produtos considerados para análise

Base do IPHMR da classe a que pertence a disposição genérica "h"

Fator de constância do resíduo gerado no equipamento genérico "j", para o produto genérico "k"

Constância do resíduo ponderada entre todos os equipamentos onde o mesmo é gerado, e para todos os produtos considerados para análise

Fator de correção para valores positivos do resíduo

Fator de correção para valores negativos do resíduo

Valor unitário do resíduo corrigido

Valor total do resíduo corrigido

Número de perguntas para análise por riscos cuja resposta é "Em potencial"

Peso da pergunta da análise por riscos no equipamento genérico "j", para o produto genérico "k"

Risco global do resíduo

Número de perguntas da análise por facilidade de minimização

Peso da pergunta da análise por facilidade de minimização do resíduo no equipamento genérico "j", para o produto genérico "k"

Custo para minimizar a geração do resíduo proveniente do equipamento genérico "j", para o produto genérico "k"

Facilidade de minimização global do Resíduo

\section{$\delta^{+}$}

$\delta$

$\$$

$\$_{\text {Total }}$

q

$\mathrm{Q}_{\mathrm{jk}}$

$\mathrm{R}$

$\mathrm{f}$

$\mathrm{F}_{\mathrm{jk}}$

$\mathrm{CM}_{\mathrm{jk}}$

F

Fonte: Adaptado de Cercal, 2000.

Para tratar a disposição final dada aos resíduos Cercal (2000) desenvolveu 25 classes englobando a natureza e a localidade do destino final, a existência ou não de um beneficiamento antes da disposição final e a função, ou seja, a utilização do resíduo seja como matéria-prima, utilidade ou sub-produto. Na Tabela 2 apresentam-se a natureza, o beneficiamento e a função da disposição final, representada por algarismos e, representada por letras, a localidade desta disposi- ção final. A partir desta tabela será feita a identificação da classe do resíduo para que posteriormente possa ser valorado, de acordo com a Tabela 3.

Análise do Resíduo por Valor: considera os aspectos ambientais (legislaçôes pertinentes) e técnico. Neste tópico o resíduo mais prioritário, ou seja, aquele que deve ser escolhido para se iniciar a minimização é o que apresentar o menor valor global. Logo, o valor global quando positivo representa lucro que a em- presa está tendo com o resíduo e quando negativo representa prejuízo, então, a disposição final deste resíduo não é adequada do ponto de vista, não só econômico, mas também ambiental e técnico (Cercal, 2000). As equações 1 a 16 calculam a análise do resíduo por valor e estão apresentadas na Tabela 4.

Análise do Resíduo por Riscos: considera os aspectos de riscos que a geração dos resíduos traz para os funcionários, vizinhos e população em geral. Para tan- 
Tabela 2 - Características das classes de disposição de resíduos

\begin{tabular}{|c|c|c|}
\hline Letra & Algarismo & Características \\
\hline & 1 & $\begin{array}{l}\text { Reutilização direta do resíduo com os materiais } \\
\text { em sua função original }\end{array}$ \\
\hline & 2 & $\begin{array}{l}\text { Reutilização direta do resíduo com os materiais } \\
\text { em função diferente da original }\end{array}$ \\
\hline & 3 & $\begin{array}{l}\text { Reutilização do resíduo após beneficiamento, com } \\
\text { os materiais em sua função original }\end{array}$ \\
\hline & 4 & $\begin{array}{l}\text { Reutilização do resíduo após beneficiamento, com } \\
\text { os materiais em função diferente da original }\end{array}$ \\
\hline & 5 & Disposição final adequada \\
\hline & 6 & Disposição final inadequada \\
\hline & 7 & Resíduo sem disposição definida. \\
\hline A & & $\mathrm{Na}$ fonte \\
\hline B & & $\begin{array}{l}\text { Em outro equipamento na mesma unidade } \\
\text { produtiva }\end{array}$ \\
\hline $\mathrm{C}$ & & Em outra unidade produtiva na mesma fábrica \\
\hline $\mathrm{D}$ & & Em outra fábrica \\
\hline E & & $\begin{array}{l}\text { Em outra fábrica, porém com beneficiamento na } \\
\text { unidade fabril onde o resíduo é gerado }\end{array}$ \\
\hline
\end{tabular}

Fonte: Adaptado de Cercal, 2000.

Tabela 3 - Análise do resíduo por valor: parâmetros matemáticos das classes de disposição

\begin{tabular}{|c|c|c|c|c|c|c|c|}
\hline Classe & $\mathrm{D}^{\mathrm{S} / \mathrm{N}}{ }_{{ }_{\phi+}}$ & $\mathrm{D}_{\$ \mathrm{~B}}^{\mathrm{S} / \mathrm{N}}$ & $\mathrm{D}^{S / N}-T_{T}$ & $\mathrm{D}_{S / \mathrm{TD}}^{\mathrm{S}}$ & $\mathrm{D}^{\mathrm{S} / \mathrm{N}}{ }_{S \mathrm{GP}}$ & $\mathrm{D}_{S / \mathrm{R}}^{\mathrm{S} / \mathrm{N}+}$ & $\xi_{\mathrm{B}}$ \\
\hline $1-\mathrm{A}$ & 1 & 0 & 0 & 0 & 0 & 0 & $+1,00$ \\
\hline $1-B$ & 1 & 0 & 0 & 0 & 1 & 0 & $+0,97$ \\
\hline $1-\mathrm{C}$ & 0 & 0 & 0 & 0 & 1 & 1 & $+0,95$ \\
\hline $1-\mathrm{D}$ & 0 & 0 & $0 / 1^{5}$ & 0 & 1 & 1 & $+0,92$ \\
\hline $2-A$ & 0 & 0 & 0 & 0 & 0 & 1 & $+0,90$ \\
\hline $2-B$ & 0 & 0 & 0 & 0 & 1 & 1 & $+0,87$ \\
\hline $2-\mathrm{C}$ & 0 & 0 & 0 & 0 & 1 & 1 & $+0,85$ \\
\hline $2-\mathrm{D}$ & 0 & 0 & $0 / 1$ & 0 & 1 & 1 & $+0,82$ \\
\hline $3-A$ & 1 & 1 & 0 & 0 & 0 & 0 & $+0,80$ \\
\hline $3-B$ & 1 & 1 & 0 & 0 & 1 & 0 & $+0,77$ \\
\hline $3-\mathrm{C}$ & 1 & 1 & 0 & 0 & 1 & 0 & $+0,75$ \\
\hline $3-\mathrm{D}$ & 0 & $0 / 1$ & 0 & 0 & 1 & 1 & $+0,72$ \\
\hline $3-\mathrm{E}$ & 0 & 1 & 1 & 0 & 1 & 1 & $+0,60$ \\
\hline 4-A & 0 & 1 & 0 & 0 & 1 & 1 & $+0,50$ \\
\hline $4-B$ & 0 & 1 & 0 & 0 & 1 & 1 & $+0,45$ \\
\hline 4-C & 0 & 1 & 0 & 0 & 1 & 1 & $+0,40$ \\
\hline 4-D & 0 & 0 & $0 / 1$ & 0 & 1 & 1 & $+0,20$ \\
\hline $4-E$ & 0 & 1 & $0 / 1$ & 0 & 1 & 1 & ZERO \\
\hline $5-A$ & 0 & 0 & 1 & 1 & 1 & 0 & $-0,20$ \\
\hline $5-B$ & 0 & 0 & 0 & 1 & 1 & 0 & $-0,40$ \\
\hline $5-\mathrm{C}$ & 0 & 0 & 1 & 1 & 1 & 0 & $-0,60$ \\
\hline 6-A & 0 & 0 & 1 & 1 & 1 & 0 & $-1,00$ \\
\hline $6-B$ & 0 & 0 & 0 & 1 & 1 & 0 & $-1,20$ \\
\hline $6-\mathrm{C}$ & 0 & 0 & 1 & 1 & 1 & 0 & $-1,40$ \\
\hline 7 & 0 & 0 & 0 & 0 & 1 & 0 & $-1,80$ \\
\hline
\end{tabular}

Fonte: Adaptado de Cercal, 2000. 
Tabela 4 - Equações para a priorização dos resíduos

\begin{tabular}{ccc}
\hline Descrição & Equação & Número \\
\hline
\end{tabular}

Equações da análise por valor

Valor unitário do resíduo

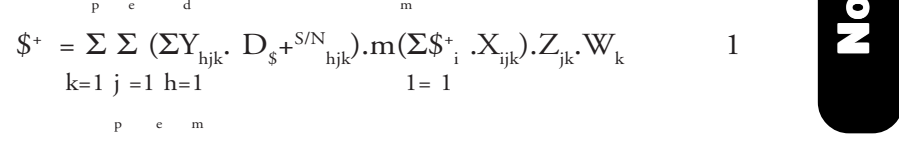

Alteração percentual admissível para o valor unitário do resíduo

$$
\begin{aligned}
& \Delta \$^{\%}=\sum \sum \sum\left(\Delta \$ \% \mathrm{i} \cdot \mathrm{X}_{\mathrm{ijk}}\right) \cdot Z_{\mathrm{jk}} \cdot \mathrm{W}_{\mathrm{k}} \\
& k=1 \quad j=1 \quad i=1 \\
& \text { p e d }
\end{aligned}
$$

Custo unitário de beneficiamento do resíduo

$$
\begin{aligned}
& \$^{-B}=\sum \sum \sum\left(Y_{h j k} \cdot D_{\$ B}^{-S / N}{ }_{h j k}^{-} \cdot \$_{B h j k}^{-}\right) \cdot Z_{j k} \cdot W_{k} \\
& k=1 j=1 h=1
\end{aligned}
$$

Custo unitário de transporte do resíduo

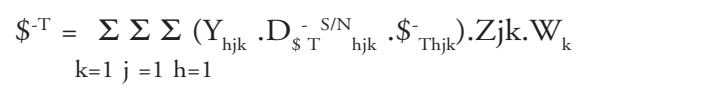

Retorno obtido conforme a disposição dada ao resíduo

Base do IPHMR

$\xi_{B}=\sum \Sigma \Sigma \xi_{B h} \cdot Y_{h j k} \cdot Z_{j k} \cdot W_{k}$ $k=1 j=1 h=1$

IPHMR

$\xi=\xi_{\mathrm{B}} \cdot \Delta \$$

Valor unitário do resíduo não corrigido

$\$^{\prime}=\$^{+}-\$_{\mathrm{B}}-\$_{\mathrm{T}}-\$_{\mathrm{TD}}-\$_{\mathrm{GP}}+\$_{\mathrm{R}}^{+}$

Constância do resíduo

$$
\underset{k=1 j=1}{K=\sum \mathrm{K}_{\mathrm{jk}}} \cdot \mathrm{Z}_{\mathrm{jk}} \cdot \mathrm{W}_{\mathrm{k}}
$$

Relação do resíduo com o processo

$\Omega=$ cte

$\delta^{+}=(1+\xi) / K \cdot \Omega \quad / / \quad \xi \neq(-1)$

$\delta^{-}=(1-\xi) \cdot \mathrm{K} \cdot \Omega \quad / / \quad \xi \neq(+1)$

Se $\$^{\prime}>0 \Leftrightarrow \$=\$^{\prime} . \delta^{+}$

Se $\$^{\prime}<0 \Leftrightarrow \$=\$^{\prime} . \delta$

Se $\$^{\prime}=0 \Leftrightarrow \$=0$

Valor total do resíduo corrigido

$\$_{\text {Total }}=\mathrm{W}_{\text {Tota }} 1 . \$$

Equações daanálise por riscos

Risco global do resíduo

$$
\begin{aligned}
& \mathrm{R}=(\Sigma \Sigma \Sigma \mathrm{Qjk} . \text { Zjk. Wk }), \Pi \\
& k=1 \quad j=1 \quad q=1
\end{aligned}
$$

Equações da análise por facilidade de minimização

Facilidade de minimização global do resíduo para $\Sigma \mathrm{F}_{\mathrm{jk}}>0$
$\mathrm{F}=\Sigma \Sigma\left(\Sigma \mathrm{Fjk}\right.$ X CMjk).Zjk. Wk se $\Sigma \mathrm{F}_{\mathrm{jk}}>0$ $k=1 j=1 f=1$ 18 P e f

Facilidade de minimização global do resíduo para $\Sigma \mathrm{F}_{\mathrm{jk}}<0$

$\mathrm{F}=\sum \sum\left(\Sigma \mathrm{Fjk} \div \mathrm{CM}_{\mathrm{jk}}\right) \cdot \mathrm{Z}_{\mathrm{jk}} \cdot \mathrm{W}_{\mathrm{k}}$ se $\Sigma \mathrm{F}_{\mathrm{jk}}<0$

Fonte: Adaptado de Cercal, 2000. 
to, são feitas uma série de quatro perguntas:

1. Existem dados reais ou estimados referentes ao resíduo?

2. Existe relação com a ocorrência de danos à saúde humana?

3. Existe relação com a ocorrência de reclamaçôes de moradores vizinhos?

4. Existe relação com a ocorrência de penalidades aplicadas (ou aplicáveis) por instituições públicas (Cercal, 2000)?

Para a pergunta 1 as possíveis respostas são SIM ou NÃO e para as demais as respostas são "já ocorreu", "em potencial" ou "isento". Se uma das perguntas 2, 3 ou 4 tiver a resposta "já ocorreu" o resíduo é diretamente classificado como prioritário. Para as respostas "não" e "isento" o modelo atribui o peso ZERO e para as respostas "em potencial" o peso é tabelado (Cercal, 2000). A análise por riscos segue a equação 17 , apresentada da $\mathrm{Ta}$ bela 4 e na Tabela 6 estão os respectivos parâmetros matemáticos. $\mathrm{Na}$ análise do resíduo por riscos quanto maior for o valor obtido, mais prioritário é o resíduo.

Análise do Residuo por Facilidade de Minimização: onde são considerados a disponibilidade de recursos técnicos, humanos e financeiros para a minimização. Assim como no caso de análise por riscos a análise por facilidade de minimização é feita com base em perguntas, porém aqui são aceitas apenas as respostas SIM ou NÃO e os pesos também são diferenciados. $\mathrm{Na}$ Tabela 7 tem-se as questôes com os respectivos pesos. Como os pesos da variável Custo de Minimização são maiores para os custos mais altos, temos um somatório positivo das perguntas, então multiplicamos pelo custo. Em contrapartida, se temos um somatório negativo das perguntas dividimos pelo custo (Cercal, 2000). Neste caso quanto menor for o valor obtido mais fácil será para minimizá-lo. A análise por facilidade de minimização segue as equações 18 e 19 da Tabela 4.

Na seqüência estão apresentadas as Tabelas 5 a 7 , propostas por Cercal (2000) que contém os parâmetros matemáticos a serem utilizados no modelo matemático.

Análise global do Residuo: Após ter sido feita a valoração dos resíduos de acordo com as equações propostas, deve-se realizar a análise global dos mesmos. Cada empresa deve decidir qual dos fatores é mais importante de acordo com a sua realidade e suas necessidades. Sugere-se que o valor global de priorização do resíduo seja feito de acordo com a Equação 20,

Tabela 5 - Análise do resíduo por valor: parâmetros matemáticos gerais

\begin{tabular}{ccccc}
\hline Relação com o processo & $\Omega$ & Constância & $\mathrm{K}$ & $\Delta \$^{\%}$ \\
\hline Intrínseco & 0,8 & Fixo & 1,1 & Máximo $=\infty$ \\
Semi-intrínseco & 1,0 & Semi-fixo & 1,0 & Mínimo $=50$ \\
Extrínseco & 1,2 & Variável & 0,9 & \\
\hline
\end{tabular}

Fonte: Adaptado de Cercal, 2000.

Tabela 6 - Análise do resíduo por riscos: parâmetros matemáticos

\begin{tabular}{cccc}
\hline Classificação do resíduo & $\Pi$ & Perguntas & $\mathrm{Q}_{\mathrm{jk}}$ \\
\hline Classe I (Perigoso) & 1 & Existem dados? & --- \\
Classe II (Não-inerte) & 2 & Danos à saúde? & 4 \\
Classe III (Inerte) & 3 & Reclamações de vizinhos? & 2 \\
& & Penalidades? & 1 \\
\hline
\end{tabular}

Fonte: Adaptado de Cercal, 2000.

Tabela 7 - Análise do resíduo por facilidade de minimização: questões e parâmetros matemáticos

\begin{tabular}{cccc}
\hline Questão & Peso & Custo & CMjk \\
\hline Parar equipamento? & 01 & Muito alto & 4 \\
Parar processo? & 02 & Alto & 3 \\
Parar unidade? & 03 & Baixo & 2 \\
Modificar equipamento? & 02 & Muito baixo & 1 \\
Modificar processo? & 04 & & \\
Modificar unidade? & 06 & & \\
Implantar equipamento? & 04 & & \\
Implantar processo? & 08 & & \\
Implantar unidade? & 12 & \\
Tecnologia disponível? & $-10,1$ & \\
Mão de Obra disponível? & $-7,1$ & \\
Recursos disponíveis? & $-15,1$ & \\
\hline
\end{tabular}

Fonte: Adaptado de Cercal, 2000.

onde A, B e C são os pesos atribuídos para cada aspecto avaliado. Assim, o valor global do resíduo deve ser multiplicado pelo peso A, o risco global deve ser multiplicado pelo peso $\mathrm{B}$ e a facilidade global de minimização pelo peso C.

Equação 20 - Valor global de priorização do resíduo

$$
\mathrm{G}=\$_{\text {Total }} * \mathrm{~A}+\mathrm{R} * \mathrm{~B}+\mathrm{F} * \mathrm{C}
$$

\section{Proposição de alternativas de minimização de resíduos}

Os resíduos foram selecionados, a partir da análise global, para que fossem feitas as sugestôes de alternativas de minimização. Estas respeitaram a hierarquia de minimização de resíduos, procurando obedecer a ordem de primeiramente evitar a geração do resíduo, depois minimizar na fonte, seguindo-se a reciclagem do resíduo e por fim o tratamento ou disposição do mesmo. Considerou-se também as práticas mais comuns de minimização de resíduos, evitando-se o uso de tecnologias caras ou complicadas e as alternativas mais práticas para a indústria.

\section{RESULTADOS E DISCUSSÃO}

A Tabela 8 contém a quantidade de resíduos encontrados por setor. Os resul- 
tados da caracterização são apresentados em conjunto com os resultados de priorização dos resíduos, uma vez que os dados necessários para a priorização são os mesmos que compóem a caracterização dos mesmos.

Resultados da Análise por Valor: os resíduos que obtiveram maior classificação pela análise por valor são quatro do setor 06 - empacotamento, três do setor 08 -ETE, dois do setor 03 - antecâmara de batatas e um do setor 05 - processo produtivo, linha 03 - extrusado de farinha de trigo Z. Mais detalhadamente, no setor $06,36,36 \%$ dos seus resíduos estão classificados entre os dez primeiros. No setor 08 , este valor aumenta para $75 \%$, dos quatro resíduos existentes neste setor, apenas o resíduo "embalagem de papel kraft" não está classificado entre os dez mais prioritários. Cabe ressaltar que os demais resíduos deste setor ("lodo primário", "lodo secundário" e "peles e pedaços de batatas, areia e gordura") estão classificados, respectivamente, em primeiro, segundo e terceiro lugar.

O resíduos classificados entre a $10^{\mathrm{a}} \mathrm{e}$ 16 a posições são todos do tipo "raspa”. A empresa produz quinze tipos de raspas. Destas a que apresentou pior classificação foi a "raspa com aroma”, ficando as demais localizadas entre $10^{\circ}$ e $36^{\circ}$ lugares.

Resultados da Análise por Riscos: de acordo com uma pesquisa realizada, $90 \%$ do resíduos produzidos não apresentam riscos à saúde (El-Fadel et al, 2001). No caso da empresa estudada por este projeto, dos 92 resíduos 64 têm risco zero, ou seja $69,56 \%$ não apresentam risco algum à saúde. Esta diferença de valores é certamente explicada devido ao fato da pesquisa realizada por El-Fadel et al (2001), não tratar de um tipo específico de indústria, sendo um levantamento geral para diversos tipos de organizações e este trabalho ser específico para a indústria de alimentos em questão.

Entre os dez prioritários para análise por riscos tem-se cinco resíduos do setor 05 - processo produtivo, três resíduos do setor 08 - ETE, um resíduo do setor 03 - antecâmara de batatas e um do setor 06-empacotamento. Assim como na análise por valor, três dos quatro resíduos do setor 08 - ETE, apresentam-se entre os dez mais prioritários, o que também acontece com o resíduo "lodo do sedimentador" gerado no setor 03 antecâmara de batatas.

Resultados da Análise por Facilidade de Minimização: dos 92 resíduos $48,91 \%$ apresentam $\mathrm{F}=-16,15$, o que

Tabela 8-Quantidade de resíduos encontrados por setor

\begin{tabular}{lc}
\hline \multicolumn{1}{c}{ Setor produtivo } & Número de resíduos \\
\hline 01 - Doca e almoxarifado de matéria-prima básica & 02 \\
02 - Doca e almoxarifado de matéria-prima para embalagem & 06 \\
03 - Antecâmara de batatas & 04
\end{tabular}

04 - Sala de mescla

05 - Processo produtivo

Linha de extrusado de farinha de milho $\mathrm{X}$

Linha de extrusado de farinha de milho $\mathrm{Y}$

Linha de extrusado de farinha de trigo $\mathrm{Z}$

Linha de extrusado de farinha de trigo W

Linha de batata chips

Linha de batata palha

Linha de preparado de farinha de trigo

06 - Empacotamento

07 - Empacotamento de sortidos

08 - Estação de tratamento de águas residuárias

Total

significa que para minimizar este resíduo depende-se de treinamento e de exigir melhor qualidade do fornecedor, $16,30 \%$ tem o $F=-24,3$ o que significa que um simples ajuste no equipamento já reduziria o resíduo. Observa-se que a maior parte dos resíduos poderia ser minimizada sem a necessidade de altos investimentos.

Entre os dez resíduos mais prioritários de acordo com a análise de facilidade tem-se quatro resíduos do setor 06-empacotamento, dois do setor 02 doca e almoxarifado de matéria-prima de embalagem, dois do setor 05 - processo produtivo, um do setor 04 - sala de mescla e um do setor 08 - ETE. Observouse uma grande diferença entre os dez prioritários das análises de valor e riscos.

Análise Global dos Residuos: foram realizadas duas análises globais. Na primeira, foram atribuídos os seguintes pesos: três para análise por valor, nove para risco e um para a análise por facilidade. Os resultados obtidos não apresentaram interação e por esta razão realizou-se a segunda análise global. Assim, atribuiu-se os seguintes pesos: para a análise por valor peso dois, análise por riscos peso três e análise por facilidade peso um. Nesta segunda análise global verificou-se uma maior interação entre os resultados obtidos e a partir dela decidiu-se quais seriam os resíduos prioritários.

\section{Alternativas de minimização de resíduos}

Para os resíduos classificados como prioritários foram propostas alternativas de minimização. A Tabela 9 contém as características destes resíduos e na seqüência apresentam-se as alternativas de minimização encontradas.

Batatas pequenas - Caso fosse feita a venda deste resíduo, para a fabricação de ração animal, o lucro anual seria de $\mathrm{R} \$ 13.642,00$, sem gastos de beneficiamento e transporte, considerando o valor estimado de $\mathrm{R} \$ 0,20 / \mathrm{kg}$ de produto. Este valor foi obtido pela média entre os valores fornecidos por duas indústrias de alimentos da Região Metropolitana de Curitiba.

Água de recirculação - Deve-se verificar com o fornecedor de batatas a possibilidade de enviar um produto mais limpo, com menos terra, assim se reduziria o número de vezes que a água de recirculação seria trocada. Outra ação que pode ser aplicada é a reutilização da água empregada na limpeza das batatas já descascadas na canaleta de recirculação, esta água possui uma qualidade aceitável para ser utilizada na limpeza primária da matéria-prima.

Scrap cru - Assim como as batatas pequenas, este resíduo é doado. Consi- 
Tabela 9 - Características dos resíduos a serem minimizados

\begin{tabular}{|c|c|c|c|c|c|c|}
\hline Resíduo & $\begin{array}{l}\text { Setor de } \\
\text { Geração }\end{array}$ & $\begin{array}{l}\text { Quantidade } \\
\text { Kg/ano }\end{array}$ & Valor & Risco & $\begin{array}{l}\text { Facilidade de } \\
\text { minimização }\end{array}$ & $\begin{array}{l}\text { Destino } \\
\text { atual }\end{array}$ \\
\hline Batatas pequenas & 01 & 68.210 & -39.410 & 0,00 & $-16,15$ & Doação \\
\hline Água de recirculação & 01 & 290.580 & -1.294 .534 & 0,33 & $-16,15$ & ETE \\
\hline Scrap cru & 05 & 114.830 & -99.519 & 0,00 & $-0,53$ & Doação \\
\hline Raspa sem aroma & 05 & 243.660 & -638.413 & 0,67 & $-22,83$ & Doação \\
\hline Raspa com aroma & 05 & 87.298 & -458.104 & 0,67 & $-20,80$ & Doação \\
\hline $\begin{array}{l}\text { Raspa com aroma do } \\
\text { caracol }\end{array}$ & 05 & 15.566 & -90.635 & 0,67 & $-29,30$ & Doação \\
\hline $\begin{array}{l}\text { Raspa com aroma setor } \\
\text { de embalagens }\end{array}$ & 06 & 180.568 & -1.547 .107 & 0,00 & $-32,30$ & Doação \\
\hline Tubetes de BOPP & 06 & 16.780 & -247.127 & 0,00 & $-5,55$ & Reciclagem \\
\hline Filme BOPP & 06 & 57.849 & -851.968 & 0,67 & $-10,77$ & Reciclagem \\
\hline Caixas de Papelão & 06 & 1.860 .458 & -1.364 .088 & 0,00 & $-16,15$ & Reciclagem \\
\hline Lodo primário & 08 & 887.600 & -14.243 .218 & 3,50 & $-7,10$ & $\begin{array}{l}\text { Aterro } \\
\text { Sanitário }\end{array}$ \\
\hline Lodo secundário & 08 & 380.400 & -6.104 .236 & 3,50 & $-10,77$ & $\begin{array}{c}\text { Aterro } \\
\text { Sanitário }\end{array}$ \\
\hline $\begin{array}{l}\text { Peles e pedaços de } \\
\text { batatas, areia e gordura }\end{array}$ & 08 & 295.867 & -4.747 .739 & 2,50 & $-10,77$ & $\begin{array}{l}\text { Aterro } \\
\text { Sanitário }\end{array}$ \\
\hline
\end{tabular}

derando como valor de venda $0,20 \mathrm{R} \$ / \mathrm{kg}$, o mesmo que o adotado para as batatas pequenas, o lucro obtido seria de $\mathrm{R} \$ 22.966,00$ ao ano.

Raspa sem aroma - A maximização do período de produção através da minimização das trocas de aromas durante os turnos diminuiria a quantidade de produto descartado. Outra maneira de reduzir a quantidade deste resíduo, seria o uso de boas práticas de fabricação, ajustando tempos, temperatura e vazóes dos equipamentos corretamente. Se as raspas fossem vendidas por $0,20 \mathrm{R} \$ / \mathrm{kg}$, o lucro seria de 48.732,00 R \$/ano.

Raspa com aroma - As raspas com aroma referem-se às perdas obtidas junto às linhas de produção, caso estas fossem vendidas para a fabricação de ração animal, o lucro seria de 17.459,60 R \$/ano, utilizando como referência o valor de cotação das batatas pequenas. Também neste caso, as boas práticas de fabricação, com o correto ajuste dos equipamentos e a maximização do período de produção pode reduzir a geração deste resíduo.

Raspa com aroma do caracol-Este resíduo foi minimizado com o ajuste da portinhola do equipamento. Reduzindo, então, o desperdício de 15 ton de produto acabado e economizando-se $\mathrm{R} \$ 3.113,20$ ao ano. Isto comprova a importância do correto ajuste dos equipamentos na minimização de resíduos.

Raspa com aroma setor de embalagens - Para sua minimização deve-se contatar o fornecedor das embalagens, de forma que o filme da embalagem utilizado esteja dentro de padróes de especificação mais rígidos e a temperatura de selagem esteja ajustada a este filme. Pois, caso o produto não seja bem selado, ocorre a geração do resíduo. Se ao invés de doado o resíduo fosse vendido, por $\mathrm{R} \$ 0,20 / \mathrm{kg}$, o lucro obtido seria de $\mathrm{R} \$ 36.133,60$ ao ano.

Tubetes de BOPP-Deve-se verificar com o fornecedor a possibilidade de reuso do material ou a substituição por algum outro tipo de matéria-prima reutilizável. Caso este resíduo fosse vendido pelo valor de $\mathrm{R} \$ 70,00$ por tonelada, que é um valor médio para este tipo de material, o lucro obtido seria de $\mathrm{R} \$ 1.174,60$ no ano.

Filme BOPP -Verificar a especificação do material no momento do recebimento e trabalhar a causa com os fornecedores, buscando obter uma matériaprima de especificação mais rígida e constante, conscientizar os funcionários de evitar desperdícios, mantendo a temperatura de selagem correta durante o processo e realizar a troca do filme quando este estiver realmente no final, não efetu- ando trocas antecipadas, são maneiras de minimizar este resíduo.

O filme de BOPP é de difícil reciclagem, o alumínio e a grande quantidade de tinta presentes no filme formam gases no momento da extrusão para a reciclagem, impossibilitando a mesma. Para que a extrusão possa ocorrer, a mesma deve ser feita em duas etapas, existindo entre elas um equipamento para remover o gás formado. $\mathrm{Na}$ empresa em que é feita a reciclagem deste material na Região Metropolitana de Curitiba, o processo é ainda adaptado e o BOPP reciclado pode ser utilizado apenas para a fabricação de peças injetadas, como vasos de flores. Porém a mesma está instalando um equipamento que possibilita a reciclagem do BOPP na forma de filme como era originalmente, comprovando que a mesma é uma técnica vantajosa para os recicladores. O lucro obtido com a venda deste filme seria de $\mathrm{R} \$ 5.784,90$ de acordo com esta empresa

Caixas de Papelão-Deve-se trabalhar a causa com os fornecedores, uma vez que as caixas de papelāo utilizadas pela empresa deveriam durar oito viagens de transporte dos produtos e estão durando apenas duas. A venda do resíduojáéfeita coma cotação de três recicladores diferentes para a escolha daqueleque apresente a melhor proposta de compra. 
Lodo primário - A redução na fonte de geração deste resíduo depende da separação das águas de processo, ricas em amido. Para tanto deve ser feito um projeto que evite que estas águas sejam direcionadas à ETE e sim direcionadas à uma centrífuga. Esta separaria a água do amido, á água poderia ser reutilizada dentro da empresa e o amido seria seco e destinado à venda. A empresa não demonstrou interesse em desenvolver o projeto de implantação de uma centrífuga neste momento, pois o mesmo envolveria uma mudança no lay out das linhas de produção de batata chips e batata palha. Além disso, seria necessária a construção de uma área para instalação da centrífuga e recuperação do amido.

Uma outra alternativa é a utilização do lodo primário na indústria de adubos para o fornecimento de matéria orgânica à adubos organominerais. O lodo é adicionado de cal virgem para evitar o desenvolvimento de microorganismos e a geração de odores. Depois é misturado à massa de produtos químicos antes da compactação para a formação de peletes.

O lodo primário conferiu aos peletes de adubo uma melhora na resistência mecânica em vista disso foi negociado com a empresa receptora de incorporar também o lodo secundário. Foram feitos testes de compactação de adubo com os dois tipos de lodos e observou-se que o lodo secundário além de aumentar o teor de matéria orgânica do adubo, não interferiu nas qualidades proporcionadas pela inclusão do lodo primário.

Lodo secundário - Para sua redução na fonte geradora seria necessário a redução da carga orgânica a ser tratada na ETE, pois a quantidade de lodo é função direta desta carga aplicada. Para tanto, o amido teria que ter separação ainda mais eficaz e a perda de óleo nos fritadores deveria ser minimizada. Outra forma de diminuir a formação de lodo é a diminuição da relação alimento/biomassa aplicada nos tanques de aeração da ETE.

Peles e pedaços de batatas, areia e gordura-A conscientização dos funcionários com relação aos cuidados que devem ser tomados na remoção máxima da gordura dos tanques auxiliaria na redução deste resíduo, bem como no melhor funcionamento da ETE, por ser a gordura um agente que dificulta a decantação dos flocos no tratamento por lodos ativados. Entretanto este resíduo não pode ser reutilizado por apresentar uma composição bastante diversificada e já em início do processo de degradação.

\section{CONCLUSÕES}

O processo de produção de alimentos pode ser otimizado com o uso das técnicas de minimização de resíduos. Estas, além de propiciarem uma redução de gastos com matérias-primas, tratamento e disposição de resíduos, ainda vão ao encontro das novas tendências de produção, que têm o respeito ao meio ambiente como um dos fatores de maior importância para a mesma.

Durante a etapa de levantamento e caracterização dos resíduos, obteve-se um conhecimento do processo industrial e dos fatores que levavam à geração dos mesmos. O modelo matemático proposto por Cercal (2000), inicialmente utilizado apenas com a finalidade de priorizar os resíduos, apresentou-se uma excelente ferramenta para o levantamento e caracterização destes.

Dos setores industriais, o setor 05 processo produtivo, é o mais complexo e com maior quantidade de resíduos, além disso, é o principal gerador de efluentes líquidos e consequentemente lodo primário e secundário. Em seguida tem-se o setor 06 - empacotamento, devido à diversidade de materiais utilizados e a sua importância na fabricação do produto.

Mesmo com 44,57\% dos resíduos sendo vendidos e $27,17 \%$ doados, sem gastos com transporte e beneficiamento dos mesmos, tem-se apenas valores negativos na análise por valor econômico, $o$ que significa que nenhum resíduo dá lucro efetivo para a empresa. Com relação aos riscos à saúde e ao meio ambiente, os resíduos encontrados apresentaram risco zero, em $69,56 \%$ dos casos. Do total de resíduos, 48,91\% seriam minimizados com treinamento de funcionários e controle na especificação das matérias-primas.

Caso as alternativas de minimização fossem adotadas o lucro obtido seria de R\$148.986,00 ao ano, incluindo aqui a venda das batatas pequenas, scrap cru e todas as raspas por $\mathrm{R} \$ 0,20 / \mathrm{kg}$, venda dos tubetes de BOPP por 0,07 R $\$ / \mathrm{kg}$ e venda do filme de BOPP por $0,10 \mathrm{R} \$ / \mathrm{kg}$. A empresa pode continuar a colaborar com a comunidade doando o dinheiro arrecadado com a venda dos resíduos ou transformando-o em benefícios para esta.

\section{AGRADECIMENTOS}

À empresa Pepsico do Brasil Ltda, aos seus funcionários e em especial à Ivone Yung Alves, pelas informaçōes, recursos e enorme colaboração, o que viabilizou este trabalho.
À fundação Coordenação de Aperfeiçoamento de Pessoal de Nível Superior-CAPES, pela concessão da bolsa de estudos.

\section{REFERÊNCIAS}

CERCAL, S.R. Proposição de modelo matemático de seleção de prioridades de minimização de residuos industriais. Dissertação (Mestrado em Tecnologia de Alimentos) - Setor de Tecnologia Química, Universidade Federal do Paraná, Curitiba. 78f. 2000.

CRITTENDEN, B.; KOLACZKOWSKI, S Waste minimization: a practical guide. Rugby: Ichem ${ }^{\mathrm{E}}, 81$ p. 1995.

EL-FADEL, M. et al. Industrial waste management im developing countries: the case of Lebanon. Journal of Environmental Management, USA, v. 61, n. 4, p. 281-300, abril 2001.

HENNINGSSON, S.; SMITH, A.; HYDE, K. Minimizing material flows and utility use to increase profitability in the food and drink industry. Trends in food science \& technology, USA, v.12, n. 2, p. 75-82, fevereiro 2001.

MATOS, S.V.; SCHALCH, V. Alternativas de minimização de resíduos da indústria de fundição. In: $27^{\circ}$ CONGRESSO INTERAMERICANO DE INGENIERIA SANITARIA Y AMBIENTAL, 2000, Porto Alegre. Relação de Trabalhos. Porto Alegre: ABES, CD-ROM. p. 1742-1753. 2000.1.

MILES, M.P.; MUNILLA, L.S.; MCCLURG, T. The impact of ISO 14000 environmental managment standards on small and medium sized enterprises. Journal of Quality Managment, USA, v. 4, n. 1, p. 111-122, 1999.

MOTA, S. Urbanização do meio ambiente. Rio de Janeiro: ABES, 352p. 1999.

PAWLOWSKY, U. Tecnologia Limpa. Apostila do Curso de Tecnologia Limpa, Curitiba, PPGTA - UFPR, 2002.

PRADO FILHO, H.R. do. Os negócios da água $e$ do lixo. Banas Qualidade - gestão, processos e meio ambiente, São Paulo, ano XI, no 123, p. 75-78, agosto 2002 .

TIBOR, T.; FELDMAN, I. ISO 14 000: um guia para as novas normas de gestão ambiental. São Paulo: Futura, 302p. 1996.

ZBONTAR, L.; GLAVIC, P. Total site: wastewater minimization wastewater reuse and regeneration reuse. Resources, Conservation and Recycling, USA, v. 30, n. 4, p. 261-275, november 2000.

Endereço para correspondência:

Bárbara Zanicotti Leite

Assessoria de Desenvolvimento e

Pesquisa da SANEPAR -

Companhia de Saneamento do

Paraná

Rua Professor José Nogueira dos

Santos, $n^{\circ} 591$ - Hauer

81630 - I20 - Curitiba - PR - Brasil

Tel.: (4I) 276-6747

E-mail: barbarazl@sanepar.com.br 